\author{
Krassen Nedeltchev \\ Urs Fischer \\ Marcel Arnold \\ Liliane Kappeler \\ Heinrich P. Mattle
}

\section{Low awareness of transient ischemic attacks and risk factors of stroke in a Swiss urban community}

Received: 13 November 2006

Received in revised form: 15 March 2006

Accepted: 28 March 2006

Published online: 2 March 2007
K. Nedeltchev · U. Fischer · M. Arnold

L. Kappeler · H. P. Mattle, MD ( $\square)$

Department of Neurology,

Inselspital

University of Bern

Freiburgstrasse 10

3010 Bern, Switzerland

Tel.: +41-31-632 3332

Fax: +41-31-632 0321

E-Mail: heinrich.mattle@insel.ch

\section{Abstract Background and} purpose Time delays from stroke onset to arrival at the hospital are the main obstacles for widespread use of thrombolysis. In order to decrease the delays, educational campaigns try to inform the general public how to act optimally in case of stroke. To determine the content of such a campaign, we assessed the stroke knowledge in our population. Methods The stroke knowledge was studied by means of a closed-ended questionnaire. 422 randomly chosen inhabitants of Bern, Switzerland, were interviewed. Results The knowledge of stroke warning signs (WS) was classified as good in $64.7 \%$. A good knowledge of stroke risk factors (RF) was noted in $6.4 \% .4 .2 \%$ knew both the WS and the RF of stroke indicating a very good global knowledge of stroke. Only $8.3 \%$ recognized TIA as symptoms of stroke resolving within 24 hours, and only $2.8 \%$ identified TIA as a disease requiring immediate medical help. In multivariate analysis being a woman, advancing age, and having an afflicted relative were associated with a good knowledge of WS ( $\mathrm{p}=0.048, \mathrm{p}<0.001$ and $\mathrm{p}=0.043$ ). Good knowledge of RF was related to university education $(\mathrm{p}<0.001)$. The good knowledge of TIA did not depend on age, sex, level of education or having an afflicted relative. Conclusions The study brings to light relevant deficits of stroke knowledge in our population. A small number of participants could recognize TIA as stroke related symptoms resolving completely within 24 hours. Only a third of the surveyed persons would seek immediate medical help in case of TIA. The information obtained will be used in the development of future educational campaigns.

Key words health education . risk factors' knowledge · stroke $\cdot$ TIA $\cdot$ warning signs

\section{Introduction}

Thrombolysis has been shown to reduce death or dependency after stroke $[1,2,3]$. However, thrombolysis can be administered safely only within the first 3 to 6 hours after stroke onset, and delay is a major barrier to its widespread use. At present, less than a third of the patients arrive within 3 hours of symptom onset and are potentially amenable to treatment. [4]

Optimizing the in-hospital stroke management can shorten door to needle time $[5,6]$. However, bringing stroke victims earlier to the hospitals would save additional time and increase the number of 
treatable patients even more [7]. Programs to improve awareness of patients with stroke to seek medical help immediately may reduce unnecessary delays to hospital arrival [8]. However, educational activities can be efficient only when the existing public perception of stroke warning sings and symptoms is considered. In order to assess the information needs of our population we performed a survey in the catchment area of our hospital.

\section{Subjects and methods}

\section{Population}

Bern is the fourth largest city and the capital of Switzerland. In May 2004, 128,000 people were living in the city and an additional 300,000 in the surroundings of Bern. $48.6 \%$ were male, $17.3 \%$ children (0 to 15 years), $40.4 \%$ young adults (16 to 44 years), $25 \%$ middle aged adults ( 45 to 64 years), and $17.3 \%$ were elderly people ( $>65$ years). The life expectancy in this population was 77 years for men and 82.5 years for women. In 2000 , cardiovascular disease was responsible for $43.3 \%$ of deaths in women and $36.2 \%$ in men.

The baseline knowledge of stroke was studied by means of a closed-ended questionnaire (see appendix). From 23 June, 2004 to 27 June 2004, 422 face-to-face interviews were performed in the streets of Bern with randomly chosen people. The survey was designed by neurologists. It was carried out with the help of medical students who were specially trained with the questionnaire and were given instructions to survey: 1) only German speaking residents of Bern and surroundings, 2) as many men as women, 3) an equal proportion of persons younger and older than 45 years.

\section{Questionnaire}

The questionnaire consists of 17 sections (see appendix):

1. Demographic data (age, sex, highest level of education). 2. The public perception of stroke among other diseases of community concern. 3 . The familiarity with the term "stroke" and the possible sources of information. 4. The location of stroke in the human body. 5. The recognition of "transient ischemic attack (TIA)" (The knowledge of TIA is assessed by yes/no questions, while some purposely false statements are included in order to detect blind guessing.) 6. The knowledge of the term "Streifung" that is used by German-speaking people to refer to stroke and to TIA. 7. The recognition of stroke warning signs (WS). $8-9$. The perception of stroke severity and whether stroke is amenable to treatment If stroke is considered treatable, an additional question referred to where the best appropriate treatment can be given. $10-$ 11. The treatment - seeking behavior is assessed, in terms of how long the interviewed person would wait until seeking medical help and where to turn for assistance. 12. Assessment of the behavior assuming the symptoms would disappear within 24 hours. $13-14$. The knowledge of stroke risk factors (RF). The subject was lso asked whether and how stroke risk can be modified. 15. Stroke victims among relatives or friends. 16. Need for further information on stroke. 17. Additional remarks or comments.

\section{Definitions}

Knowledge of the stroke WS and RF was considered as good according to the definitions proposed by Montaner and co-workers [9]
- Good knowledge of stroke WS: Those who recognize correctly three or more symptoms of the list and make one error or less.

- Good knowledge of stroke RF: They can name five or more and make one error or less.

- Very good global knowledge of stroke: Those who recognise both stroke WS and RF.

In addition, we defined:

- Good knowledge of TIA: They recognize TIA as symptoms related to stroke that recover completely within 24 hours, identify TIA as potentially harmful and would seek medical help immediately.

\section{Statistical analysis}

Nominal and ordinal variables were expressed as frequencies. The associations between them were analyzed using the Pearson's $\chi^{2}$ test. The associations between age and the nominal variables was assessed using the Mann-Whitney test. A multiple logistic regression analysis was used to identify factors independently associated with a good knowledge of WS and RF of stroke/TIA. p value $<0.05$ was considered significant. All analyses were performed using the SPSS statistical software.

\section{Results}

Four hundred and twenty two persons ( 177 men and 245 women) were surveyed. Figure 1 shows their distribution of age, sex and highest level of education.

When asked about the most devastating disease according to their opinion, $33 \%$ of the surveyed people nominated cancer, $15 \%$ AIDS, $13 \%$ stroke, $12 \%$ myocardial infarction, $10 \%$ Alzheimer's dementia, and $8 \%$ depressive disorders. The remaining $9 \%$ named diabetes mellitus, rheumatic and lung diseases.

Ninety-seven percent of the surveyed people had already got some information about stroke, mostly from television $(40 \%)$, periodicals $(33 \%)$, radio $(14 \%)$, the family physician $(8.6 \%)$, lectures $(4.5 \%)$, or the internet $(3.1 \%)$ Some subjects indicated more than one source of informat Younger age was associated with television as information source $(p=0.01)$. With advancing age, persons were more likely to receive the pertinent information from the family physician $(p<0.049)$. There were no significant associations between the remaining sources of information and age, sex or level of education.

Ninety one percent of the surveyed people located stroke and TIAs in the brain. Seven percent considered the heart as the site of strokes, $1 \%$ the skull and $1 \%$ the spinal cord.

Three hundred and sixty eight subjects (87.2\%) could not recall to have ever heard the term transient ischemic attack (TIA). Thirty five persons (8.3\%) recognized TIA as stroke related symptoms resolving completely within 24 hours, $10(2.4 \%)$ as a mild 
Fig. 1 Age, sex and level of education of the surveyed people $(n=422)$

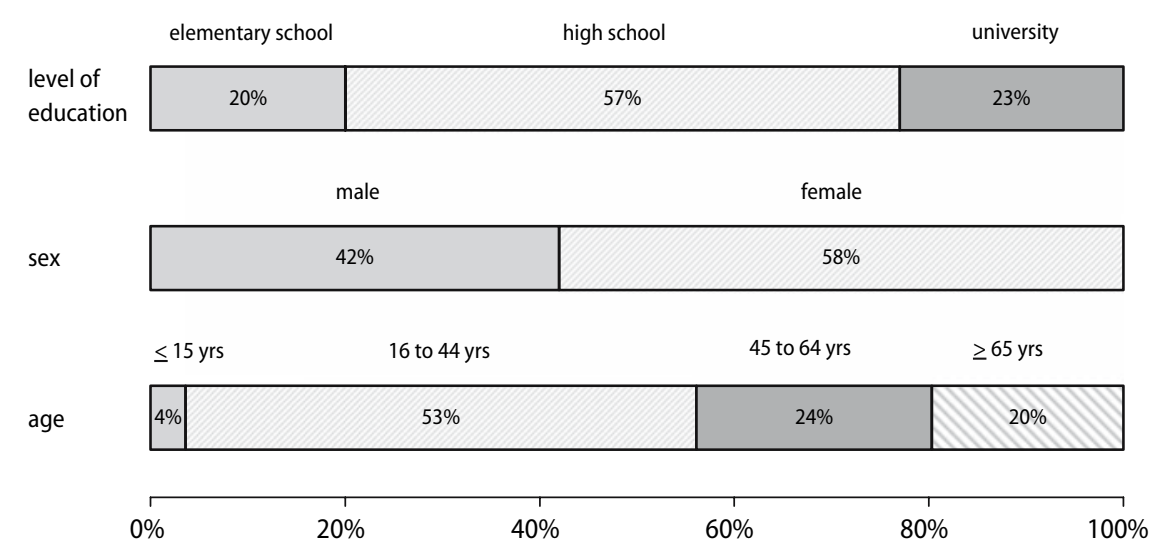

Table 1 Knowledge of TIA, stroke WS and RF in respect of sex, level of education and age

\begin{tabular}{|c|c|c|c|c|c|c|c|c|c|}
\hline \multirow[t]{2}{*}{-} & \multicolumn{2}{|l|}{ Sex } & \multicolumn{4}{|c|}{ Level of education } & \multicolumn{3}{|l|}{ Age } \\
\hline & Male & Female & Primary school & High school & University & $\leq 15$ years & $16-44$ years & $45-64$ years & $\geq 65$ years \\
\hline Good knowledge of stroke warning signs & $101(57)$ & $171(70)^{*}$ & $48(58)$ & $159(66)$ & $64(66)$ & $5(33)$ & $127(57)$ & $79(79)$ & $61(73) \dagger$ \\
\hline Good knowledge of risk factors of stroke & $14(8)$ & $13(5,3)$ & $4(4,9)$ & $6(2,6)$ & $17(17,9) \dagger$ & 0 & $16(7,2)$ & $5(4,9)$ & $6(7,2)$ \\
\hline Good knowledge of TIA & $4(2,2)$ & $8(3,2)$ & $2(2,5)$ & $5(2,2)$ & $5(5,6)$ & 0 & $10(4,5)$ & $1(1)$ & $1(1,2)$ \\
\hline Knowledge of the word "Streifung" & $165(93)$ & $225(92)$ & $73(88)$ & $225(93)$ & $92(95)$ & $11(66)$ & $201(90)$ & $95(95)$ & $83(92)$ \\
\hline
\end{tabular}

Data are given as number of surveyed people (\%). ${ }^{*} \mathrm{p}=0.005$, difference between groups (Pearson $\chi^{2}$ test); $\dagger p<0.001$, difference between groups (Pearson $\chi^{2}$ test)

Table 2 Prevalence of correctly recognized stroke WS and RF

\begin{tabular}{lllllllll}
\hline Number & 0 & 1 & 2 & $\vdots$ & 3 & 4 & 5 \\
\hline Prevalence of correctly recognized WS & $1 \%$ & $1 \%$ & $4 \%$ & $\vdots$ & 6 & $11 \%$ & $21 \%$ \\
Prevalence of correctly recognized RF & $22 \%$ & $18 \%$ & $17 \%$ & $\vdots$ & $4 \%$ & $2 \%$ & $0.5 \%$ & $0.5 \%$ \\
\hline
\end{tabular}

The dotted line indicate the cut-off point between poor and good knowledge (correct recognition of 3 and more stroke WS or RF by making 1 or less error) of stroke WS and RF

stroke, and $9(2.1 \%)$ as a heart disease. Of the 35 persons who recognized TIA as stroke related symptoms that recover within 24 hours, only $12(2.8 \%)$ identified it as a potentially harmful disease and would have sought medical help immediately, i.e. had a good knowledge of TIA. Good knowledge of TIA did not depend on age, sex, level of education or having a stroke victim among relatives or friends (Table 1).

Three hundred and ninety subjects (93\%) indicated that they had heard the term "Streifung". There were no associations between the knowledge of the word "Streifung" and the age, sex and level of education (Table 1). Sixty three percent would use the term to refer to a mild stroke, $22 \%$ to a transient ischemic attack (TIA), and $7 \%$ to a stroke of whatever severity.

The knowledge of stroke WS was good in 272 persons (64\%) (Tables 1 and 2). In a multiple logistic regression analysis, being a woman, advancing age, and having a stroke victim among relatives and friends were independently associated with a good knowledge of stroke WS $(\mathrm{p}=0.048, \mathrm{p}<0.001$ and $\mathrm{p}=0.043$ ).

Good knowledge of RF was noted in 27 surveyed persons (6.4\%) (Tables 1 and 2). Their knowledge was associated with university education ( $\mathrm{p}<=0.001$ ), but not with age, sex or having an afflicted relative or friend. Eighteen of the 422 surveyed persons (4.2\%) recognised both the WS and the RF of stroke and thus had a very good global knowledge of stroke.

The recognition of stroke as a disease that can be treated increases with advancing age $(p=0.01)$. In the event of symptoms suggesting stroke, $64.4 \%$ of the participants would seek medical help immediately (emergency medical systems (EMS), nearest hospital or nearest stroke center), $35.6 \%$ would wait until the 
symptoms disappear spontaneously or would arrange a visit with the family physician. Older people are more likely to call the family physician $(p=0.049)$ and younger persons the EMS $(p=0.049)$. Participants with a higher level of education are more likely to call the EMS ( $40 \%$ elementary school, $49 \%$ higher school, $60 \%$ university; $\mathrm{p}=0.018$ ). If the symptoms resolved spontaneously within 24 hours (TIA), 58\% would call the family physician to arrange a visit within the next days or weeks, $18 \%$ would seek immediate help in the nearest hospital, and $16 \%$ would call the EMS. Eight percent would ignore the problem and would not seek medical help.

\section{Discussion}

The majority of Swiss people have one or two vascular risk factors, overweight and hypertension being most common by far [10]. Good knowledge of RF and modification of these two RF would reduce the incidence of stroke significantly. On the other hand, rapid recognition of and reaction to stroke WS through immediate use of the EMS, priority transport with prenotification of the receiving hospital, rapid and accurate diagnosis and treatment at the hospital are components of the so called "stroke chain of survival" [11]. Education campaigns that propagate efficient treatment-seeking behavior are therefore the first and important step to strengthen the chain of survival. To reach this target and to bring the correct message appropriately, assessment of the public needs for information should precede the development and implementation of educational campaigns for the public [12].

However, there are no data regarding the public perception of stroke in Switzerland, and the crosspopulation comparability of the few studies from other industrialized European countries is somewhat questionable $[9,13,14,15]$.

A study conducted among 1000 visitors of several primary health centers in Barcelona, Spain, revealed a good knowledge of stroke WS in 50\% and a good knowledge of the RF in $37 \%$ of the surveyed persons [9]. We used a similar questionnaire and the same definitions of good knowledge of stroke WS and RF, so that a direct cross-population comparison between two European cities can be made. Similar to Barcelona, the majority of the participants in Bern belonged to the middle class urban population. Nevertheless, the recognition of stroke RF was considerably lower in our population, while the good knowledge of WS was about the same as in Spain. In addition, one fifth of the surveyed Spanish participants had a very good knowledge of the disease, while the proportion of persons with a very good knowledge of stroke in Bern was $4.2 \%$. Whether the variations between Barcelona and Bern are due to knowledge deficits only, or other factors, e.g. participant's selection (visitors of primary health centers in Barcelona, randomly chosen inhabitants of Bern in the present study), might have played a role, is open.

Several studies have assessed the public awareness of stroke in different parts of the world. In view of the variations in study design and surveyed population, it is uncertain whether the results are applicable more generally. In addition, the public awareness of a disease seems to vary considerably with the time. In 1997, Kothari et al. assessed the awareness of stroke in U.S. patients who presented at the ED with a potential stroke [16]. Of 163 patients who were able to complete the questionnaire 63 patients (39\%) did not know a single sign or symptom of stroke. Similarly, $43 \%$ of patients did not know a single RF for stroke. A recent analysis of the public awareness of stroke and the knowledge of the proper emergency response in 17 U.S. states and the U.S. Virgin Islands indicated that public awareness of several stroke signs is high (up to $94.1 \%$ ), but the ability to recognize the five major WS was low (19.6\%). A more complex and updated image of the public perception of stroke can only be obtained, if studies from different countries of the world implement standardized instruments of assessment. An adequate description of the background population (urban or rural, age and sex distribution, level of education, income etc.) is also a prerequisite.

In Switzerland, the primary place to go for medical help is the family physician. In this survey, younger people and those with a higher level of education were more likely to call the EMS in the event of symptoms suggesting stroke, while older people would call their family doctor. An unpublished analysis of all referrals of stroke patients to our institution showed that calling the family physician resulted in significant delays from symptom onset to arrival at the hospital. Therefore, information campaigns to propagate calling the EMS in the event of stroke would significantly speed up "the stroke chain of survival". A surprising and alarming finding of our study is the very high ignorance of transient ischemic attacks. Only $2.8 \%$ of the study participants related correctly the definition of TIA and would seek medical help instantly in the event of spontaneous recovery of the symptoms. For comparison, $8.2 \%$ of $10^{\prime} 112$ U.S. adults new the definition of TIA and $8.6 \%$ could identify a typical symptom [17]; In the USA, deficits in TIA knowledge were particularly common in young adults, men, and those with lower income and less education. In our population, sex, age, level of education and having an afflicted relative did not influence the knowledge of TIA. The German term "Streifung" contributes 
further to the terminological uncertainty and alters the knowledge of TIA, since it is used to refer both to a mild stroke and a TIA.

One large study has specifically evaluated the attitude adopted in the event of TIA [9]. In this study, $53.5 \%$ of the surveyed persons would contact the EMS in the event of a TIA, while $42.5 \%$ would go to the family doctor and $4 \%$ would ignore it completely. For comparison, $34 \%$ of our people would call the EMS or go to the nearest hospital, 58\% would call their family physician and $8 \%$ would not take any action. Given the high prevalence of TIA and its associated risk of stroke, information campaigns focusing on TIA awareness could have a major impact on public health $[11,18,19]$.

There are few studies assessing the impact of the mass-media on the public awareness of a health issue $[20,21,22]$. A recent study compared the effectiveness of advertising in print and on television to increase the ability to name two or more WS of stroke [23]. Television advertising increased the mean number of WS named, the percentage who could name two or more WS, and the percentage naming 4 of the 5 . Television increased the knowledge of both men and women and of people with lower education but not of people older than 65 years. In our population, younger age was associated significantly with television as a source of information, and television reached all demographic groups irrespective of sex, age and education.

Our study has some limitations. First, female participants are slightly overrepresented in the present survey $(58 \%)$. Second, we did not keep tabs on subjects who refused to participate. These are potential sources of bias that cannot be ignored in the analysis of the results of the study.

To summarize, the present survey reveals significant gaps in the Swiss people's knowledge of stroke warning signs and risk factors. A small number of participants could recognize TIA as stroke related symptoms resolving completely within 24 hours. The German term "Streifung", although familiar to most participants, is used to refer both to a mild stroke and a TIA and thus leads to a terminological uncertainty. Only a third of the surveyed persons would seek immediate medical help in case of TIA. The information obtained in this survey will be used to develop our next educational campaign that will try to fill in the specific gaps in stroke knowledge in our population.

\footnotetext{
Acknowledgements The authors thank all the volunteers who helped to survey the kind people of Bern and its surroundings. This study was supported by a grant of the Stroke Division of the Swiss Heart Foundation.Conflict of Interest: None declared.
}

\section{Appendix}

\section{The Bernese Survey of Public Knowledge of Stroke}

1. Personal data: a) sex (male, female); b) age; c) highest level of education.

2. Which of the following diseases is of utmost importance for you? (AIDS, cancer, stroke, Alzheimer's dementia, myocardial infarction, diabetes mellitus, depression, rheumatoid arthritis, lung diseases)

3. Have you ever heard about stroke? If yes, quote one of the following sources of information: periodicals, television, radio, lecture, family physician, internet, other.

4. Where is the disease located? (brain, arm and leg, heart, skull, spinal cord)

5. Have you ever heard about "transient ischemic attack (TIA)"? If yes, please specify what does TIA mean: (a heart disease, mild stroke, symptoms of stroke that recover completely within 24 hours.

6. Have you ever heard the term "Streifung"? If yes, what would you use the term Streifung to refer to? (mild stroke, TIA, stroke of whatever severity)

7. I will mention a number of symptoms. Do you know which of them are manifestations or symptoms of stroke (loss of strength or numbness of one half of the body and/or of the face, difficulties in speaking or understanding, sudden unconsciousness, high blood pressure, chest pain, sudden and severe headache, sudden loss of vision, gait disturbances, epileptic seizures).

8. Is stroke in your opinion a severe or a harmless disease?

9. Is stroke in your opinion a treatable disease? If yes, where, respectively who should treat it? (family physician, every hospital, specialized stroke center)

10. How long can be waited until thrombolysis, i.e. clot-busting therapy? (3 weeks, 48 hours, 24 hours, 3 hours)

11. I will mention a number of health care providers, which can be called in the event of symptoms suggesting stroke. Which one would you contact? (family physician, EMS, the nearest hospital, the nearest specialized hospital, I would wait symptoms disappear spontaneously)

12. What would you do if you were confident that the symptoms will disappear spontaneously within 24 hours? ( go to your family physician, call EMS, go to the nearest hospital, the nearest specialized hospital, take no further action)

13. Which one of the following health care providers would you call in the event of symptoms or signs of stroke, if you knew that symptoms will 
disappear spontaneously within the next 24 hours? (family physician, EMS, the nearest hospital, the nearest specialized hospital, I would wait symptoms to disappear spontaneously).

14. I will mention some diseases and medical conditions. Could you tell me which ones predispose an individual to suffer a stroke, i.e. are risk factors of stroke (high and/or low blood pressure, tobacco, alcohol, low physical activity, overweight, myocardial infarction, cardiac arrhythmia, diabetes mellitus, epilepsy).
15. Is it possible to modify or even to reduce the risk of stroke? If yes, how? (control of overweight, blood pressure, physical activity, healthy nutrition, smoking cessation).

16. Has any of your relatives or acquaintances ever suffered a stroke?

17. Would you like to receive further information on stroke?

18. Remarks.

\section{References}

1. National Institute of Neurological Disorders and Stroke rt-PA (1995) Stroke Study Group. Tissue plasminogen activator for acute ischemic stroke. $\mathrm{N}$ Engl J Med 333:1581-1587

2. Furlan A, Higashida R, Wechsler L, Gent M, Rowley H, Kase C, Pessin M, Ahuja A, Callahan F, Clark MW, Silver F, Rivera F (1999) for the PROACT investigators. Intra-arterial prourokinase for acute ischemic stroke: the PROACT II study: a randomized controlled trial: Prolyse in Acute Cerebral Thromboembolism. JAMA 282:200320011

3. Wardlaw J, Berge E, Del Zoppo G, Yamaguchi T (2004) Thrombolysis for acute ischemic stroke. Stroke 35:29142915

4. Harraf F, Sharma AK, Brown MM, Lees KR, Vass RI, Kalra L (2002) A multicentre observational study of presentation and early assessment of acute stroke. BMJ 325:17

5. Chapman KM, Woolfenden AR, Graeb D, Johnston DC, Beckman J, Schulzer M, Teal PA (2000) Intravenous tissue plasminogen activator for acute ischemic stroke: A Canadian hospital's experience. Stroke 31:2920-2924

6. Nedeltchev K, Arnold M, Brekenfeld C, Isenegger J, Remonda L, Schroth G, Mattle HP (2003) Pre- and In-Hospital Delays from Stroke Onset to Intraarterial Thrombolysis. Stroke 34:12301234

7. Vanhooren G, Davalos A, Fieschi C, Grond M, Hacke W, Kaste M, Von Kummer R, Larrue V, Lees K, Wardlaw J, Wahlgren N (2004) First year of SITS monitoring study (SITS-MOST) on broad implementation of thrombolysis in stroke: Wide differences in delays between onset-treatment, door-imaging and door-needle. Cerebrovasc Dis 17(suppl 5):108
8. Rossnagel K, Jungehulsing GJ, Nolte $\mathrm{CH}$, Muller-Nordhorn J, Roll S, Wegscheider K, Villringer A, Willich SN (2004) Out-of-hospital delays in patients with acute stroke. Ann Emerg Med 44:476-483

9. Montaner J, Vidal C, Molina C, Alvares-Sabin J (2001) Selecting the target and the message for a stroke public education campaign: a local survey conducted by neurologists. Eur J Epidemiol 17:581-586

10. Nedeltchev K, Arnold M, Baumgartner R, Devuyst G, Erne P, Hayoz D, Sztajzel R, Tettenborn B, Mattle HP (2005) on behalf of the Swiss Heart Foundation and the Cerebrovascular Working Group of Switzerland. Vascular risk factors in the Swiss population. J Neurol 252:1210-1216

11. Adams HP Jr, Adams RJ, Brott T, del Zoppo GJ, Furlan A, Goldstein LB, Grubb RL, Higashida R, Kidwell C, Kwiatkowski TG, Marler JR (2003) Hademenos. Guidelines for the early management of patients with ischemic stroke: A scientific statement from the stroke council of the American Stroke Association. Stroke 34:1056-1083

12. Bartholomew LK, Parcel GS, Kok G (1998) Intervention mapping: a process for developing theory- and evidencebased health education programs. Health Educ Behav 25:545-563

13. Derex L, Adeleine P, Nighoghossian N, Honnorat J, Trouillas P (2004) Knowledge about stroke in patients admitted in a French Stroke Unite. Rev Neurol (Paris) 160:331-337

14. Carroll C, Hobart J, Fox C, Teare L, Gibson J (2004) Stroke in Devon: knowledge was good, but action was poor. J Neurol Neurosurg Psychiatry 75:567-571
15. Parahoo K, Thompson K, Cooper M, Stringer M, Ennis E, McCollam P (2003) Stroke: awareness of the signs, symptoms and risk factors-a population-based survey. Cerebrovasc Dis 16:134-140

16. Kothari R, Sauerbeck L, Jauch E, Broderick J, Brott T, Khoury J, Liu T (1997) Patients awareness of stroke signis, symptoms and risk factors. Stroke 28:1971-1875

17. Johnston SC, Fayad PB, Gorelick PB, Hanley DF, Shwayder P, van Husen D, Weiskopf T (2003) Prevalence and knowledge of transient ischemic attack among US adults. Neurology 60:14291434

18. Lovett JK, Dennis MS, Sandercock PA, Bamford J, Warlow CP, Rothwell PM (2003) Very early risk of stroke after a first transient ischemic attack. Stroke 34:e138-e140

19. Coull AJ, Lovett JK, Rothwell PM (2004) Oxford Vascular Study. Population based study of early risk of stroke after transient ischaemic attack or minor stroke: implications for public education and organisation of services. BMJ 328:326

20. Dixon H, Borland R, Segan C, Stafford H, Sindall C (1998) Public reaction to Victoria's “2 Fruit'n' 5 Veg Every Day" campaign and reported consumption of fruit and vegetables. Prev Med 27:572-581

21. McVey D, Stapleton J (2000) Can antismoking television advertising affect smoking behaviour? Controlled trial of the Health Education Authority for England's anti-smoking TV campaign. Tob Control 9:273-282

22. Bauman AE, Bellew B, Owen N, Vita P (2001) Impact of an Australian mass media campaign targeting physical activity in 1998. Am J Prev Med 21:41-47

23. Silver FL, Rubini F, Black D, Hodgson CS (2003) Advertising strategies to increase public knowledge of the warning signs of stroke. Stroke 34:1965-1968 